\title{
ROMANIZACIÓN DE RITUALES FUNERARIOS ORETANOS: LA TUMBA 1 DEL Cerro de las Cabezas (Valdepeñas, Ciudad Real)
}

\section{Romanization of Funerary Rituals in Oretania: The Tomb 1 of the Cemetery of the Cerro de las Cabezas (Valdepeñas, Ciudad Real, Spain)}

LUIS BENÍTEZ DE LUGO ENRICH ${ }^{1}$, ANTONIO MANUEL POVEDA NAVARRO², DIONISIO URBINA MARTÍNEZ ${ }^{3}$, CATALINA URQUIJO ÁLVAREZ DE TOLEDO ${ }^{3}$

(1) Dpto. de Prehistoria y Arqueología. Universidad Autónoma de Madrid. UNED, Centro asociado de Ciudad Real. luis.benitezdelugo@uam.es (2) Dpto. de Prehistoria, Arqueología, Historia Antigua, Filología Griega y Filología Latina (Área de Historia Antigua). Universidad de Alicante. antonio.poveda@ua.es (3) c/ Llano, 25.45370. Santa Cruz de la Zarza (Toledo). arqueoexperiences@gmail.com

\section{RESUMEN:}

La correcta aplicación de un procedimiento de prevención de impactos y protección al patrimonio arqueológico, desarrollado con motivo de la implantación de una línea eléctrica de alta tensión, permitió en 2013 el hallazgo de una necrópolis junto al Cerro de las Cabezas (Valdepeñas, Ciudad Real). Hasta el momento se desconocía la localización del área funeraria de este gran oppidum. Las catorce tumbas documentadas durante esta breve intervención (catorce días de trabajo) corresponden a los enterramientos de cremaciones en urnas, con piezas de ajuar asociadas como fusayolas, fíbulas, armas y recipientes cerámicos. Se presenta el estudio ceramológico de la Tumba 1; la única de entre las excavadas que cuenta con ajuar de importación. En concreto la pieza importada es un plato de t.s. itálica con sello del taller de L. Titivs, fechado entre 15/40 d.C. Es, por tanto, un enterramiento de comienzos de nuestra era. El hallazgo permite verificar que en ese momento persisten en la Oretania los ritos funerarios indígenas ibéricos, que utilizan ocasionalmente cerámicas romanas de importación.

Palabras clave: Romanización, arqueología funeraria, cremación, ajuar, cerámica terra sigillata itálica, Oretania.

Abstract:

The proper application of a method of protection and preventing impacts to Archaeological Heritage, developed because of the installation of an electrical power line, has led to the discovery of the necropolis beside Cerro de las Cabezas (Valdepeñas, Ciudad Real). The location of the burial area of this great Iron Age oppidum was unknown until now. The fourteen graves documented during this brief intervention correspond to the cremations buried in urns, with goods associated as fusayola, fibulae, weapons and pottery. In this work is presented the ceramologic study of Tomb 1. This is the only one between the graves excavated which has imported pieces. The imported object is a plate of italian terra sigillata, dated between 15/40 AD and sealed in the factory of L. Titivs. Therefore it is an early burial of our era. The finding verifies than in these years persist in Oretania indigenous Iberian funerary rites, which occasionally used import Roman pottery

Key words: Romanization, funerary archaeology, trousseau, cremation, ceramic Italian terra sigillata, Oretania. 


\section{INTRODUCCIÓN}

La obra de implantación de la Línea de Alta Tensión $132 \mathrm{kV}$ Valdepeñas-Santa Cruz de Mudela (Ciudad Real) precisó, en aplicación de la normativa vigente, de la realización de un estudio arqueológico previo al inicio de las obras, así como una vigilancia arqueológica en ciertos tramos del trazado durante la fase de movimientos de tierras.

En cumplimiento de los artículos 52.4 de la Ley 4/2013 de Patrimonio Cultural de Castilla-La Mancha y 44.1 de la Ley 16/1985 de Patrimonio Histórico Español (deber del promotor/dirección facultativa de paralizar inmediatamente los trabajos en caso de ser hallados restos de valor cultural, del tipo que fuere, durante la ejecución de una obra y comunicar el hallazgo a la Administración competente) la dirección arqueológica de los trabajos notificó el 17 de junio de 2013 a la Dirección General de Cultura la detección de evidencias arqueológicas en los Apoyos 20-2 y 20-3 de la torre 20 de la citada línea eléctrica.

En este lugar, a una cota aproximada de $-0,40 \mathrm{~m}$, fue localizada al excavar el hoyo para cimentar la torre, una asociación de cerámicas, carbones y mampuestos en posición derivada, en la ribera oriental del río Jabalón. Las obras de instalación de la línea eléctrica fueron detenidas en el Apoyo 20, quedando el lugar balizado desde ese momento. El control arqueológico siguió aplicándose al resto de la obra, que permaneció activa. La posición del Apoyo 20 fue ligeramente modificada hacia el N, con el fin de evitar la afección a las evidencias arqueológicas detectadas.

Bajo supervisión arqueológica fueron excavados sus dos nuevos apoyos septentrionales, que resultaron estériles desde el punto de vista arqueológico.

En el lugar en donde se produjeron los hallazgos arqueológicos, al S y al pie de la torre 20, fue desarrollada en agosto de 2013 una excavación por medios manuales, una vez fue emitida la correspondiente autorización administrativa (exp. Cultura 05/1613). Dicha intervención permitió hallar una necrópolis oretana de incineración.

El estudio del sitio avanzó más tarde mediante una prospección arqueológica intensiva con detector de metales del nivel superficial alrededor de las catas excavadas. Este trabajo se desarrolló en diciembre de 2013, una vez recibida la específica autorización administrativa para ello (exp. Cultura 13-1239). De esa forma fue posible recuperar del nivel superficial, tras su georreferenciación, numerosas piezas arqueológicas compatibles con la necrópolis detectada que se encontraban dispersas por la superficie de una parcela roturada e inundada recurrentemente por el río Jabalón. Esta colección se encuentra en estudio en estos momentos.

El trabajo que se presenta ahora expone los resultados del estudio acera de las características y contenido de la Tumba 1 de la necrópolis del Cerro de las Cabezas, la única de entre las detectadas que presenta material de importación.

\section{CONTEXTO ARQUEOLÓGICO}

El Cerro de las Cabezas (Valdepeñas, Ciudad Real) se encuentra ubicado en la margen izquierda del río Jabalón, en la cuenca hidrográfica del río Guadiana. El asentamiento protohistórico ocupa una superficie de unas 14 ha en un cerro que alcanza una cota de 805 m.s.n.m., con pendientes pronunciadas al $\mathrm{N}$ y $\mathrm{O}$, y suaves al E y S. Al $\mathrm{O}$ su visibilidad se encuentra limitada por una cadena de cerros. Por ello, en este flanco sus murallas no descienden hasta el valle como sucede en los flancos sur o este, sino que se mantienen más protegidas a una cota elevada del cerro, viéndose apoyadas en su defensa por una probable atalaya cuadrangular construida aproximadamente a $430 \mathrm{~m}$ de distancia sobre el cerro vecino, que se levanta enfrente, al O. Esa atalaya es una edificación con mampostería bien careada y sin evidencias de monumentalidad, pero con una clara ubicación estratégica de vigilancia. En definitiva, la ciudad, coronada con una acrópolis apreciable mediante fotografía aérea, se extiende básicamente por la ladera del cerro orientada al mediodía y, fuera de sus murallas, cuenta con elementos tan característicos del paisaje ibero como son una atalaya, al $\mathrm{O}, \mathrm{y}$ una necrópolis en la ribera opuesta del río, al E. Todos estos elementos quedaron inventariados y protegidos mediante la carta arqueológica elaborada en 2005, dentro del Ámbito de Protección Arqueológica A02-Cerro de las Cabezas. La necrópolis detectada se encuentra dentro del mencionado ámbito arqueológico, en el entorno de influencia del oppidum y en una posición clásica en relación a la que los cementerios -no sólo protohistóricos- suelen tener con respecto a las ciudades, motivo por el cual se ha denominado con el mismo topónimo (fig. 1).

Por otra parte, en su comarca la posición del asentamiento también es claramente estratégica. Primero, por estar situado en la arteria de comunicación E-O que constituye el Jabalón, con hitos de población importantes 


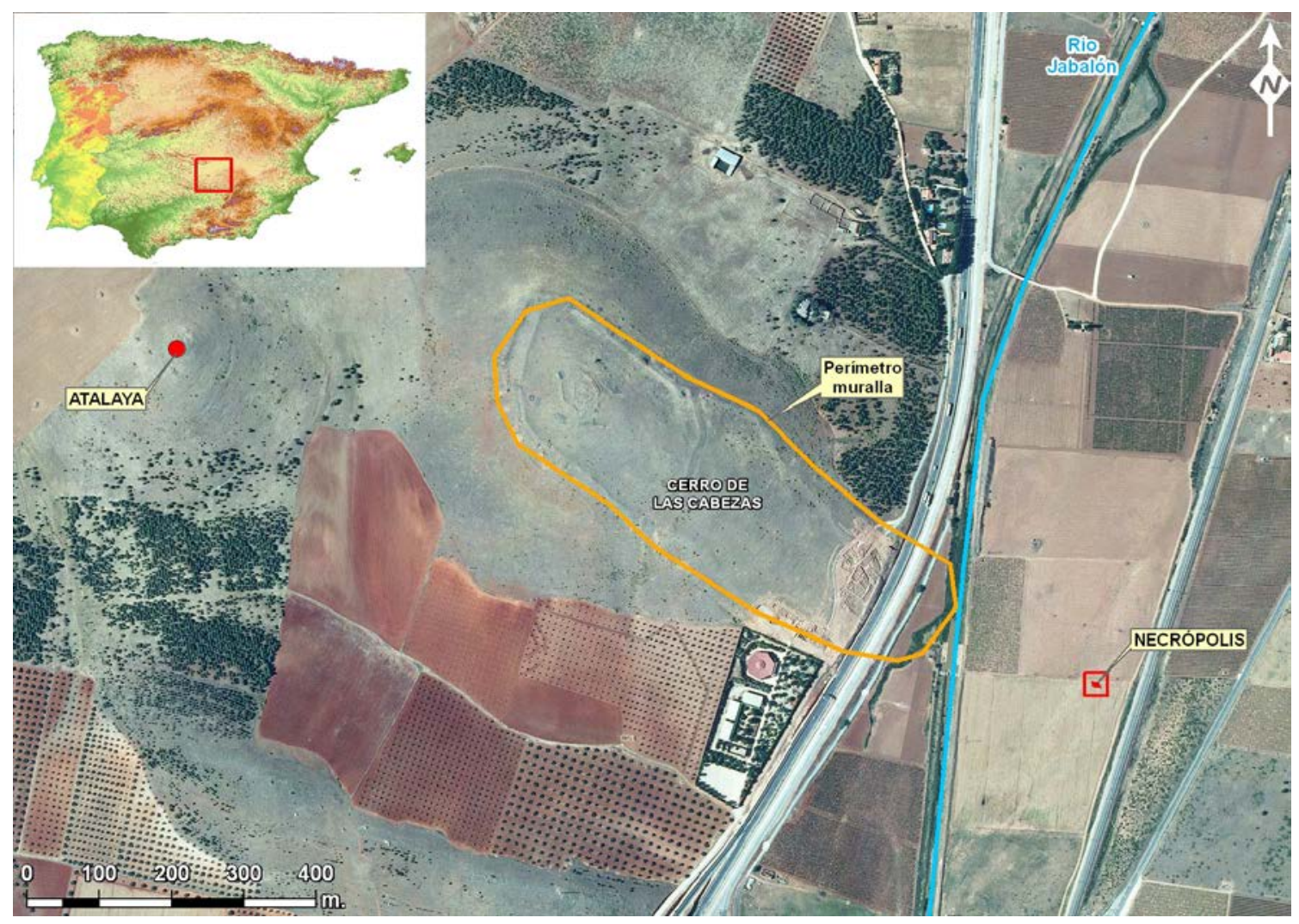

Fig. 1: Localización del Cerro de las Cabezas (Valdepeñas, Ciudad Real) y los principales elementos de su entorno. Dibujo: J. Moraleda.

próximos, como el propio Cerro Domínguez a occidente y Alcubillas a oriente. En segundo lugar, por su proximidad al paso hacia el Valle del Guadalquivir a través del río Despeñaperros, del Guarrizas o del Puerto del Muradal, con el importante hito intermedio que constituye el Santuario de Collado de los Jardines (Santa Elena, Jaén). También es significativa su proximidad al corredor natural de La Mancha, que permite la salida del mineral oretano y las relaciones hacia el N (Benítez de Lugo et al. 2004).

En el yacimiento se han realizado sucesivas campañas de excavaciones arqueológicas desde 1985, habiéndose puesto al descubierto una notable superficie del asentamiento protohistórico en la parte más baja de la ladera E. A lo largo de esas campañas de excavación en este poblado se han encontrado varios enterramientos infantiles en ambientes domésticos. Sus características concretas se desconocen, debido a que no han sido presentados aún a la comunidad científica por sus excavadores. Lo mismo sucede con los cadáveres inhumados en el acceso meridional del poblado. Se trata de los restos humanos de unos decapitados. En 2010 el director de las excavaciones arqueológicas, el arqueólogo municipal Julián Vélez Rivas, difundió que los decapitados eran dos jóvenes de entre 10 y 15 años, enterrados tras su sacrificio ritual bajo unas cornamentas de ciervo y junto con unas espadas dobladas (Benítez de Lugo y Moraleda 2013: 242). Al cabo de cuatro años se acometió un análisis antropológico de los restos. La nueva información aportada señalaba que los restos humanos pertenecían no a adolescentes, sino a dos hombres de entre 30 y 50 años, quizás ladrones o enemigos del poblado que fueron castigados y exhibidos, según difundió mediante prensa el Ayuntamiento. En todo caso se trataba de enterramientos aislados.

Con estos antecedentes, hasta el momento no se conocía ninguna necrópolis en los alrededores de este oppidum. La zona de enterramientos ahora detectada se encuentra sólo a $222 \mathrm{~m}$ al E de las murallas orientales del poblado, en una zona de vega que resulta claramente visible desde 
la ciudad. El cauce del río Jabalón separa el ámbito funerario del poblado. Esta disposición, además, sitúa al espacio funerario a favor de los vientos dominantes, que son del O. De esta forma los humos y olores de las cremaciones se dirigirían habitualmente en dirección contraria al poblado, evitándose tal vez así las molestias a lugares de habitación. Las evidencias materiales dispersas por la superficie del terreno atestiguan poblamiento alrededor de esta ciudad ibérica durante la romanización. Aunque por el momento no ha sido descubierta una fase de hábitat iberorromano en el área excavada (menos del 10\% de la superficie del poblado), dado que se han encontrado tumbas de esta época, resulta evidente que existió asentamiento coetáneo en un lugar que bien podría encontrarse en cualquiera de los lugares del poblado que aún no han sido estudiados, o bien en la vega al pie del cerro. Algo similar se ha observado, por ejemplo, en los vecinos oppida oretanos de Cerro Domínguez (Granátula de Calatrava, Ciudad Real) o Giribaile (Vilches, Jaén). Se trata, ni más ni menos, del conocido proceso de descendimiento del oppidum al llano vivido en numerosas ciudades prerromanas con motivo de la romanización. El del Bronce Final, ibérico, romano y medieval

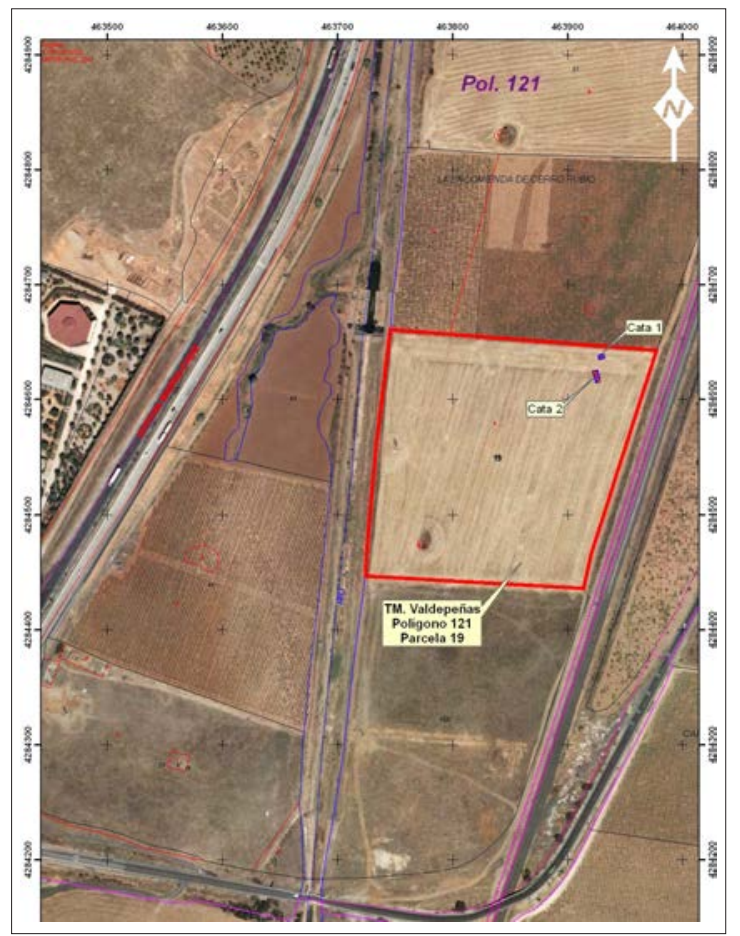

Fig. 2: Localización de las dos catas abiertas. Dibujo: J. Moraleda. no son tres yacimientos diferentes, sino fases producidas por la evolución de un mismo grupo humano. Lógicamente éste debe estudiarse de forma unitaria, junto con sus sucesivos cementerios (Urbina et al. 2015: 47 y 49).

Los trabajos arqueológicos que ahora se presentan han permitido recuperar materiales de una necrópolis de incineración en posición primaria (fig. 2). En las dos catas abiertas fueron descubiertas un total de 14 tumbas de incineración y dos construcciones claramente vinculadas con el funcionamiento de la necrópolis: un ustrinum de planta elíptica donde se quemaban los cadáveres, abundantes manchas de cenizas y un túmulo de planta cuadrada que alberga seis de las tumbas documentadas. Las demás tumbas descubiertas estaban enterradas en los alrededores de las construcciones descritas (fig. 3).

También fueron documentados dos muros que debieron formar parte de otros complejos mayores, que se prolongan probablemente más allá de los límites del área excavada. En ella no se constataron evidencias de la existencia de estructuras asociadas a hábitat en este lugar. Entre las piezas de ajuar asociadas a las tumbas destacan recipientes cerámicos, armas, fíbulas y hasta dieciocho fusayolas.

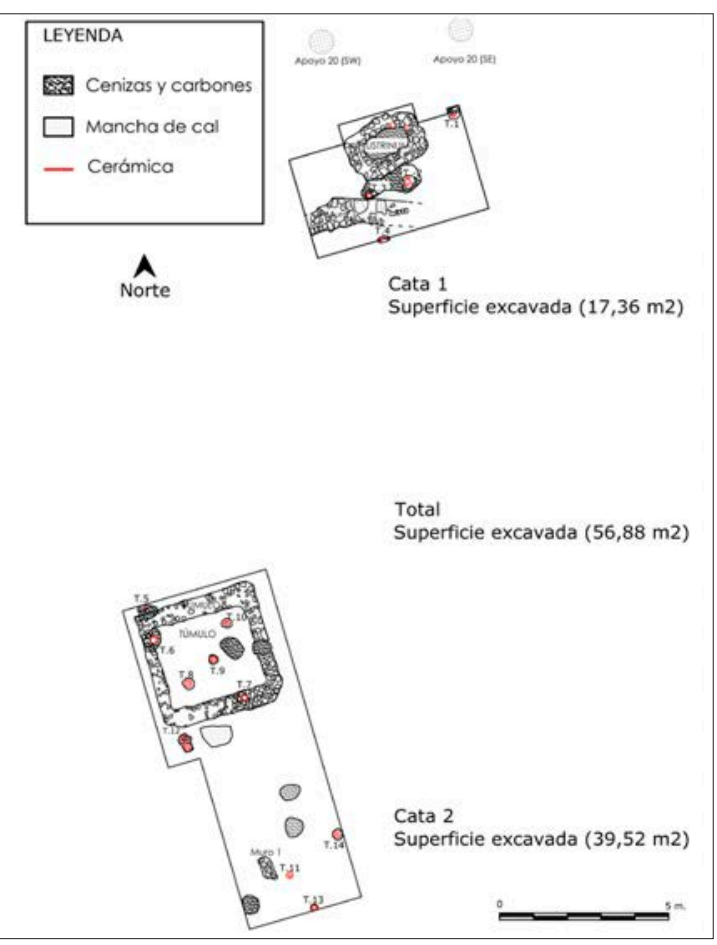

Fig. 3: Planta general de las dos catas abiertas. Dibujo de E. Mata. 


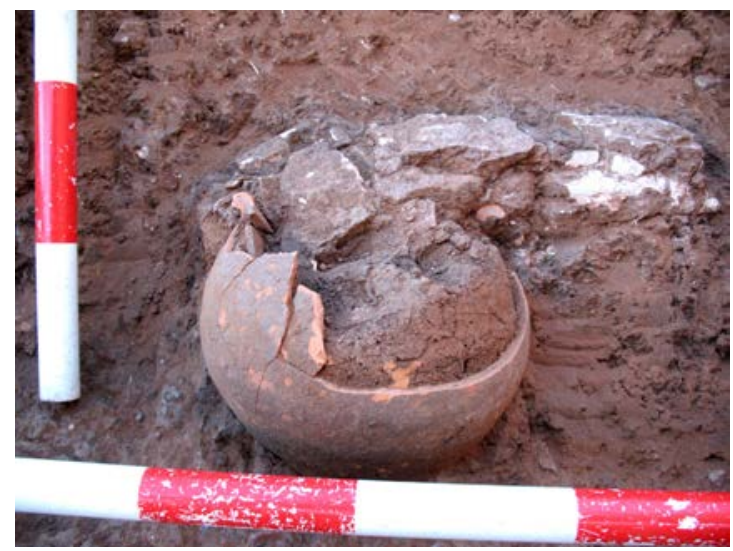

Fig. 4: Tumba 1 (UC3).

En los alrededores, fuera de las catas abiertas, fueron recuperados del nivel superficial otros objetos arqueológicos que habían sido removidos por las labores agrícolas y por las avenidas del río Jabalón. De hecho, el lugar de los hallazgos se vio sumergido por el desbordamiento del río en los tres años anteriores a nuestra intervención, pues en esta zona la corriente fluvial meandrizaba y, aunque ahora se encuentra canalizado, en el pasado se desbordaba con facilidad

La Tumba 1 (Cata 1, UC3) se encontraba directamente excavada en el suelo, aproximadamente a un metro al E del ustrinum. Consistía en un hoyo que contenía en su interior cenizas y una urna funeraria globular cubierta por un plato de terra sigillata itálica, que en su interior contenía más cenizas y pequeños fragmentos de hueso (fig. 4). Todo el conjunto aparecía en su contexto arqueológico primario sellado por pequeñas lajas de caliza cuidadamente dispuestas. La cota superior de las lajas era $-82 \mathrm{~cm}$. El plato de sigillata colocado entre las lajas y la urna fue encontrado roto y muy fragmentado (Poveda y Benítez de Lugo 2015).

Las demás tumbas encontradas fueron enterradas más superficialmente. Por ello, su parte superior desapareció, seccionada por los aperos agrícolas. No se ha detectado la cubierta o tapadera de ninguna de ellas en su posición original. Todas ellas se encuentran aún en proceso de recomposición y estudio.

A continuación se presenta el estudio correspondiente a la Tumba 1, la más íntegra y única estructura de entre las documentadas en las catas abiertas de la cual se ha recuperado material importado en un contexto funerario cerrado.

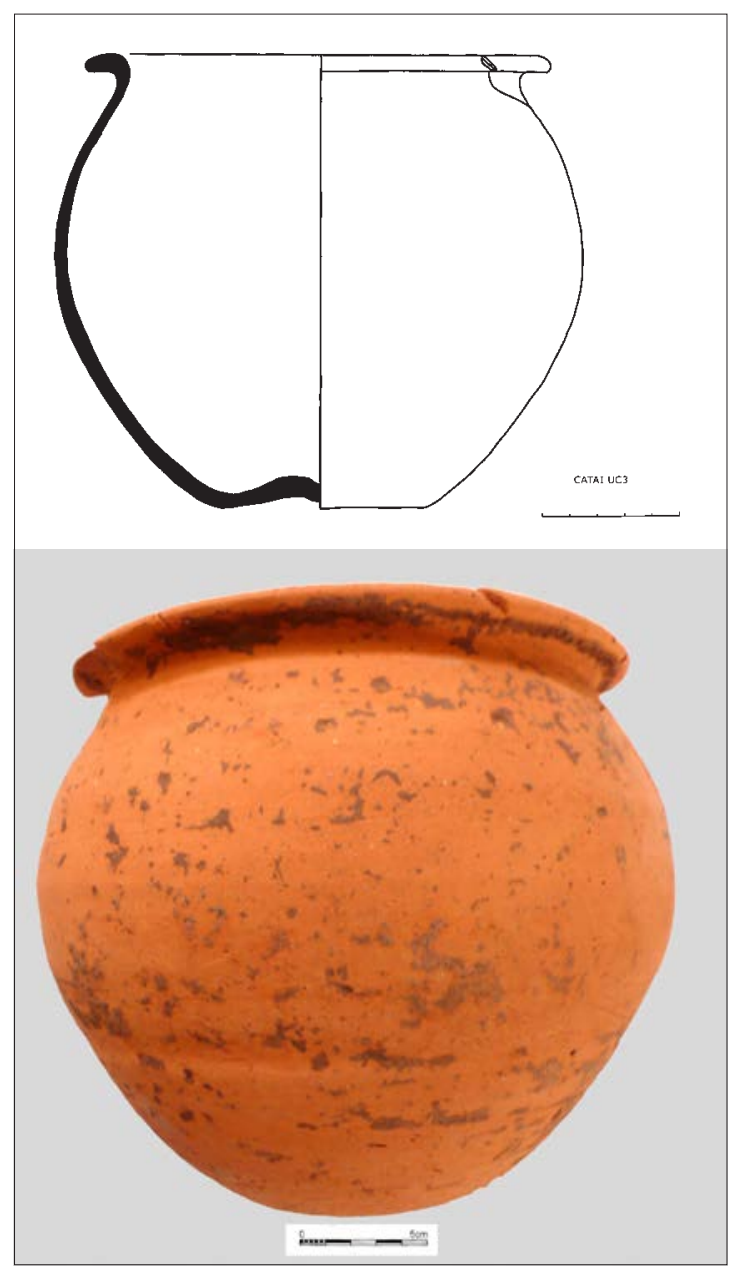

Fig. 5: Urna cineraria de la Tumba 1.

\section{MATERIALES DE LA TUMBA 1}

\section{LA URNA FUNERARIA}

La urna de la Tumba 1 corresponde a una ollita pintada de tradición indígena enmarcable dentro del tipo Abascal 18a: vaso globular con labio sencillo. Como manifiesta este autor (Abascal 1986: 109-110) se trata de la forma más común en la Meseta Sur. Parece estar imitando la forma Mayet XXb de paredes finas, fabricada desde Tiberio a Claudio (fig. 5).

No es posible clasificarla en ninguna de las variedades que distingue Abascal en función de su decoración -dos o más bandas vinosas que delimitan frisos en los que puede aparecer o no decoración (Abascal 1986: 110)-, ya 


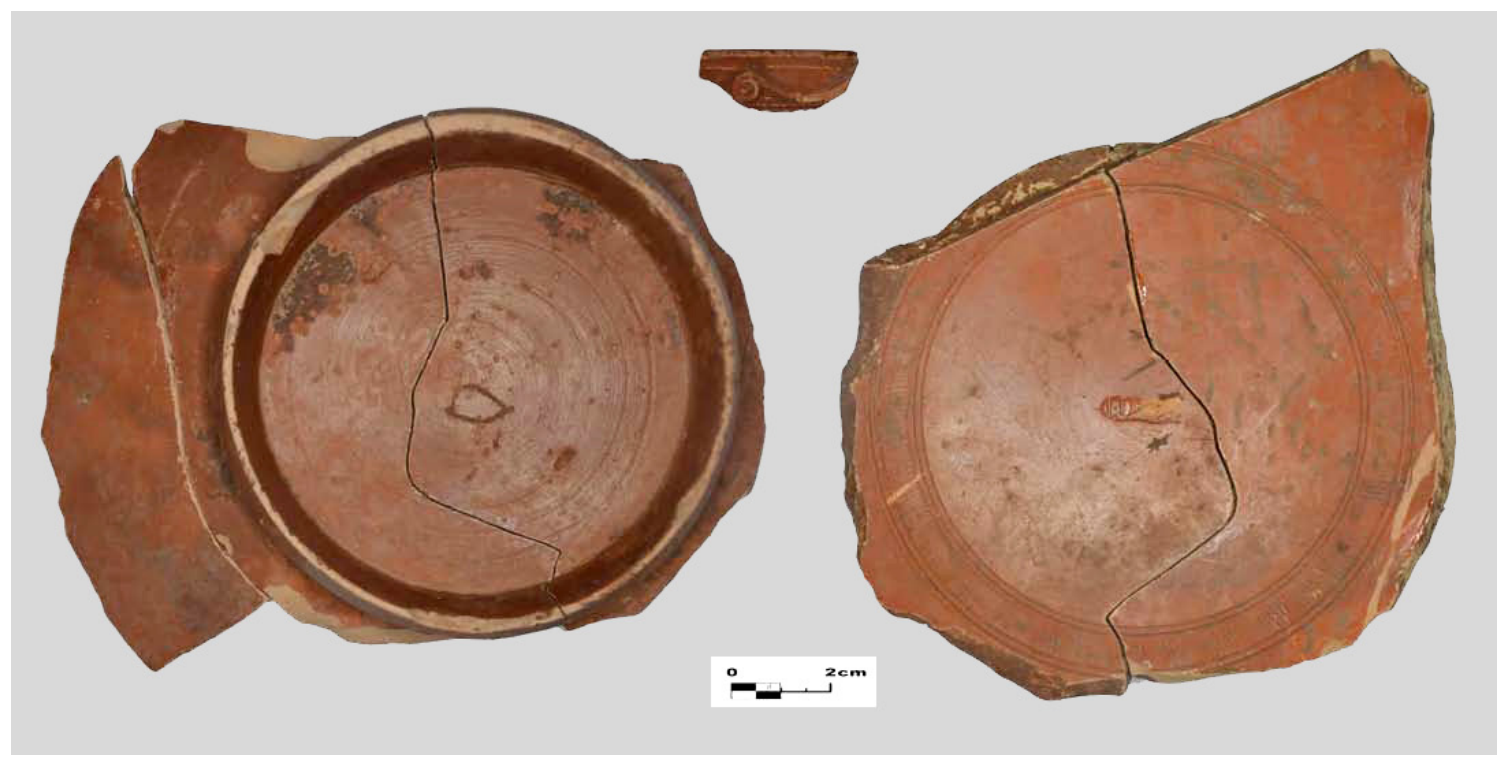

Fig. 6: Detalle del fondo (externo, a la izquierda; interno, a la derecha) del plato de TSI utilizado como tapadera de la urna funeraria.

que ésta se ha perdido por completo. Tan sólo podemos constatar que la pasta es de color rojo-anaranjado y que la superficie en donde debería estar la pintura se ha deteriorado, a buen seguro por los efectos del terreno arcilloso y el lavado producido por las recurrentes avenidas del río Jabalón que inundan el lugar.

Algo semejante ocurría con las piezas pintadas de tradición indígena halladas en Madrigueras (Carrascosa del Campo, Cuenca), en donde el terreno había alterado por completo varias urnas de pastas similares a la que nos ocupa: EI1V2 y EI5V2 (Urbina et al. 2013: 125 y ss.).

\section{LA TAPADERA}

Cubriendo la urna de tipo globular se halló en alto estado de fragmentación un plato de terra sigillata itálica, del que sólo se recuperó el 35\%. La pieza presenta unas características morfológicas propias de un plato de borde ligeramente exvasado, labio bífido con acanaladura superior, indicado al exterior (193 mm). Presenta pared externa de perfil recto y triple moldura delgada al exterior con decoración a base de motivos aplicados de dobles volutas o espirales geminadas en el friso inferior, así como pared interna de perfil convexo y ligeramente exvasada.

El fondo es de sección recta con decoración radial a ruedecilla flanqueado por dos acanaladuras. Conserva parcialmente, en la parte central interna, marca de alfarero in planta pedis con leyenda L.TITI. A su vez presenta grafito poligonal anepígrafo post cocturam en el fondo externo.

El pie de fondo está elevado y es de sección triangular, con ángulos rectos en la unión exterior del pie con el fondo y ligero engrosamiento al centro de la sección del fondo (fig. 6).

La pasta es rosada depurada, con algunas inclusiones calizas que precipitan en amarillo, rotura rugosa YR 2,5YR5/5 (Munsell 2004), barniz anaranjado ligero 2,5R4/6 de mala calidad, aunque espeso en el interior (Munsell 2004).

El perfil interno de la pared, claramente exvasado, aproxima el ejemplar recuperado a los prototipos de la subforma Conspectus 18.2.1, recordando el momento de hibridación y/o transición entre las subformas Conspectus 18.2 y la 20.5. Algo que también ocurre en la ejecución del labio bífido de este ejemplar, lo que le aproxima al labio de la subforma Conspectus 20.5.1. Sin embargo, el perfil de la pared es fiel a la subforma Conspectus 20.5.2 (Ettlinger et al. 1990: 82-86). En efecto, la triple moldura externa -el acusado engrosamiento de la unión pared/fondo por medio de un refuerzo de la moldura inferior, que además mantiene un ángulo recto y la ausencia de acanaladuras internas en la pared del platoconduce a la idea de un típico caso de combinación intencionada de elementos tomados de ambos subtipos, que coexisten en el mercado de forma paralela (fig. 7). 


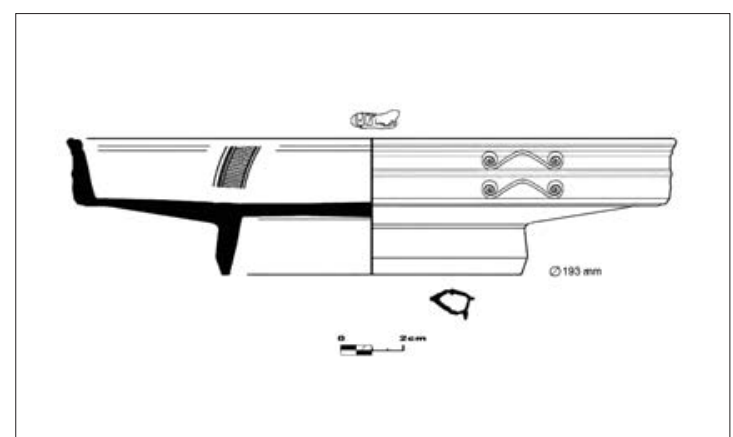

Fig. 7: Forma Consp.20.5.1-2 dispuesta como tapadera en la Tumba 1. Dibujo: Oppida.

El plato sometido a estudio presenta sigillum in p.p. de L(VCIVS)TITI(VS) CVArr.2052=2203) (Oxé et al. 2000: 445), productor establecido exclusivamente en Arezzo. Se conocen 34 nombres de alfareros tardíos asociados al nombre de L. Titivs, cuyos nomina aparecen en cartelas rectangulares sencillas o dobles, y en mayor medida in planta pedis, siendo estas últimas las de fase tardía, cronológicamente siempre posterior a los años 13/15 d.C.

El sello muestra como primera letra latina una $L$ seguida de interpunción y, después, la parte superior de las cuatro letras que constituyen la leyenda TITI. Finalmente, aparece representada la parte final del pie con una línea vertical de la que irradian los dedos hacia la derecha, que se aprecian levemente (fig. 8).

\section{DISCUSIÓN}

Platos como el ahora encontrado vienen a sustituir otro tipo de platos que habían sido utilizados anteriormente en ritos funerarios iberos, tales como los de barniz rojo, los de barniz negro áticos y sus imitadores campanienses.

La Forma 20 del plato hallado en la Tumba 1 como tapadera supone en sí misma una evolución con respecto a la Forma 18, en la que se inspira y de la que se diferencia por la desaparición de las acanaladuras internas radiales sobre el fondo. Estas acanaladuras son sustituidas, por norma general, por otra más elaborada que utiliza la técnica de la ruedecilla aplicada sobre el fondo interno, y que aparece en ocasiones flanqueada por alguna acanaladura de diferente grosor, muy similar a la pieza que se presenta (fig. 9).

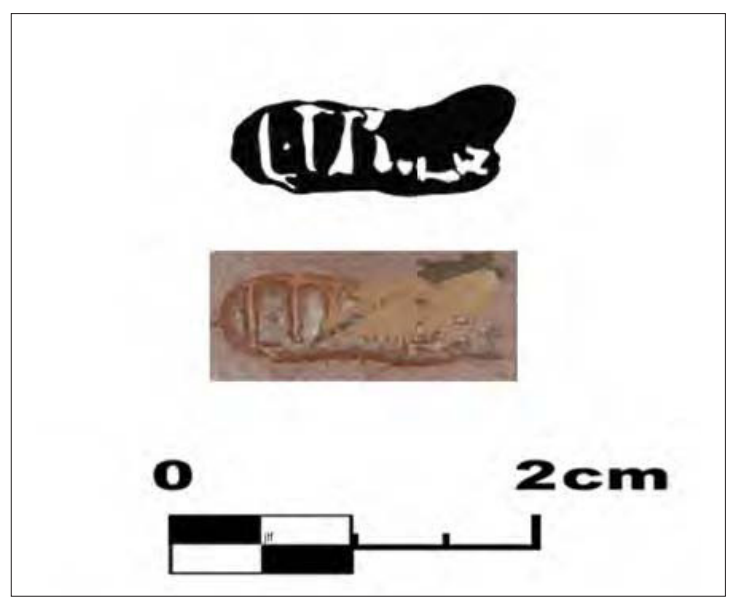

Fig. 8: Detalle del sigillvm p.p. de L.TIT(I). Dibujo: Oppida.

En concreto las subformas 20.5.1 y 20.5.2 revelan elementos innovadores con respecto a la formas 18 y 20 , lo que posibilita una datación mas precisa. En efecto, la incorporación de la técnica decorativa de la triple moldura en la superficie lisa de la pared supone una importante innovación estilística con respecto a las variantes de la forma principal. Esta técnica rompe la superficie de la pared del plato, habilitando de facto dos frisos perpendiculares que permiten la inserción de decoración con técnicas diversas como la de ruedecilla y/o motivos aplicados, que es la de nuestro plato.

Otro de los elementos que la diferencian con respecto a la Forma 18 es la presencia de decoración consistente en una doble voluta o espiral doble unida por una tira sencilla de arcilla que se aplica sobre los frisos resultantes, en parejas y perpendiculares a la moldura central. Esta corriente estilística en la decoración externa, presente en algunas subformas de la Forma 20, es perfectamente visible en uno de los fragmentos recogidos de la pieza hallada en la Tumba 1. Lo anterior, unido a la escasez de estos motivos en los diferentes fragmentos recuperados, permite afirmar que este plato contó tan sólo con cuatro motivos aplicados de espirales geminadas, dispuestas en grupos de dos, opuestas entre sí y perpendiculares sobre y bajo la moldura central. Este recurso estilístico en la decoración comienza a ponerse de moda a partir de mediados y/o finales del reinado de Tiberio, si bien tuvo abundantes perduraciones hasta los años 70 y 80 d.C.

Estos detalles, que también se aprecian en los fragmentos conservados del plato -y que por norma general se dan para toda la subforma 20.5-, caracterizan las variantes 


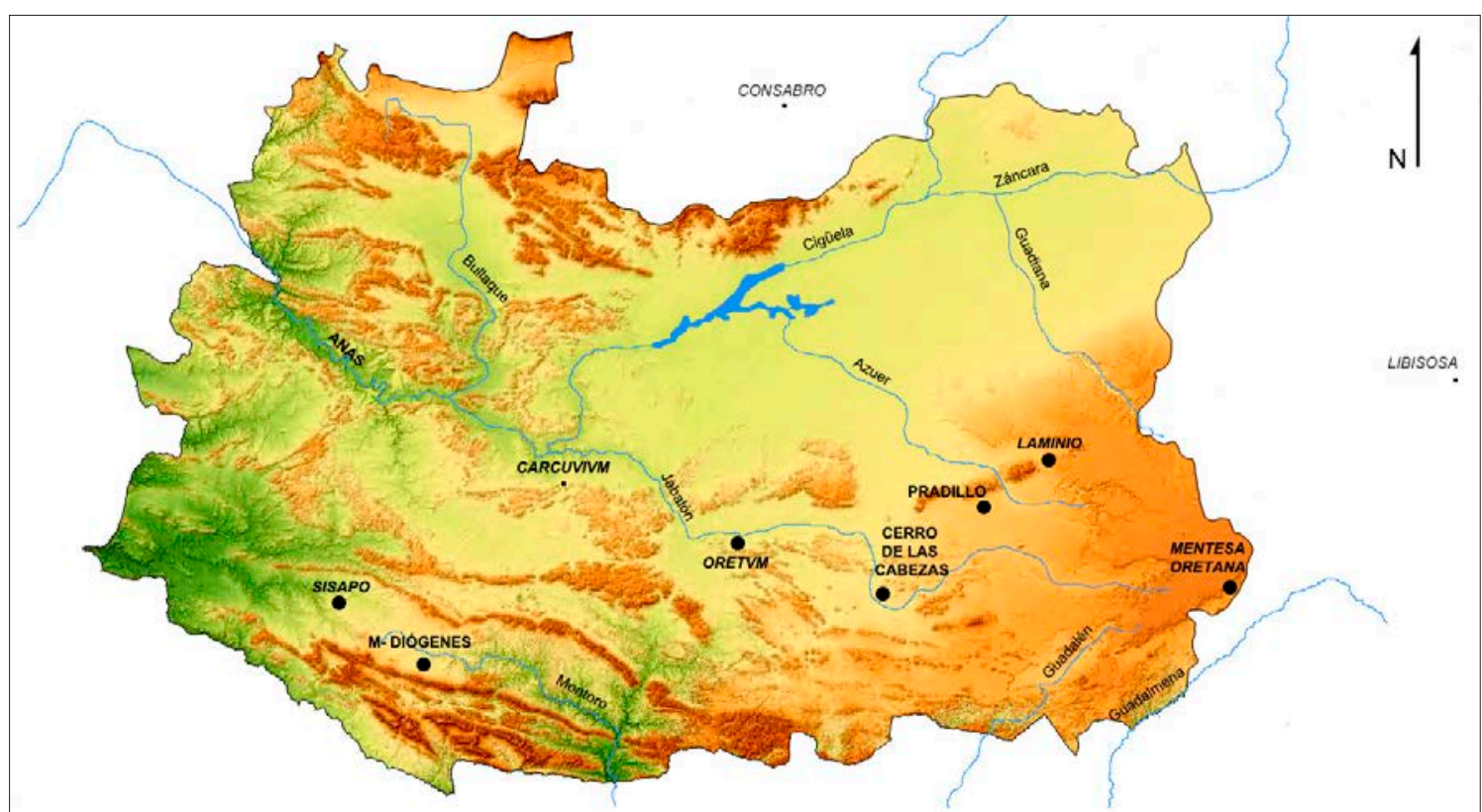

Fig. 9: Hallazgos de TSI en la provincia de Ciudad Real. Dibujo: Oppida.

de la Forma 20 por la presencia de motivos decorativos de espirales dobles o geminadas, aplicadas en paralelo en ambos frisos en los que queda distribuida la pared. En el ejemplar conservado sólo se advierte la presencia de este motivo en el friso inferior, debido a la alta fragmentación de la pieza que impide una reconstrucción íntegra de la misma.

El pie de fondo mantiene las características formales de la Forma 18, aunque es más elevado y delgado, más frecuente y propio en la subforma $20.5 \mathrm{y}$, en la pieza de sigillata importada hallada en la Tumba 1, utilizada como tapadera de la urna globular.

En cuanto a la producción de la Forma 20 y sus variantes más difundidas -como son la 20.2 y 20.5 - es posible señalar que fueron producidas en serie y se integran en un macrogrupo formal de vasos de borde recto que abarca desde la forma 18 a la 30 (Ettlinger 2002: 48).

La Forma 20, cuya producción alcanzó altos niveles de estandarización hacia el cambio de Era, fue fabricada en un amplio conjunto de talleres itálicos dedicados a la fabricación de sigillata que parece se localizaban en el Valle del Po, en una zona indeterminada de la Italia central y en la propia Arezzo (Ettlinger et al. 1990: 86). Situación que no se reproduce en apariencia con las subformas 20.5.1 y 20.5.2, cuyos indicios apuntan a producciones realizadas exclusivamente en algunos talleres aretinos, al menos en los de Lucius Gellius (Ettlinger 1990: 86) y Lucius Titius (Oxé et al. 2000: 23), por lo que su producción pudo no ser tan extensa como otras subformas o variantes de la Forma 20.

Esta forma posee similitudes estilísticas y rasgos compartidos que recuerdan a la Forma Consp. 20.1 (Pucci.10,6) (Pucci 1985: tv.121), documentada en superficie en Sisapo-La Bienvenida (Fernández-Ochoa y Zarzalejos 1991: 258), así como otros rasgos de la Forma Consp.18.2 (Pucci.10.5,7) (Pucci 1985: tv.121) que se encuentran en dos platos hallados en la necrópolis del Camino del Matadero (Alhambra, Ciudad Real), en un contexto muy similar al plato ahora analizado dado que fueron usados como tapadera de urnas funerarias de incineración en las tumbas 6, 7 y 8 (Fernández Rodríguez y Serrano 1985: 192).

Se han documentado estructuras de producción alfarera de L.Titivs en Arezzo (Carciarelle, Fonte Pozzuolo, Orciolaia, Piaggia di Murello, Piaza San Agostino, Piazza San Francesco, Santa Maria in Grandi, Teatro Petrarca (Via G. Monaco) (Olcese 2002: 9-22) y, de forma especial, en Vía dei Cenci. En esta última localización se ha documentado un establecimiento productivo compuesto por los restos de un horno y una balsa de decantación, en la que se manufacturan cerámica común y sigillata exclusivamente de L. Titivs. 
No se conocen, por otro lado, estructuras de producción asociadas a este grupo de productores fuera este núcleo alfarero, lo que permite afirmar que este plato pertenece sin duda a una producción genuina de Arretium.

El Taller de L. Titivs produce preferentemente formas lisas (Hofmann 1985: 29). Sobre muchas de ellas se conoce un buen número de nombres de esclavos asociados a este nomen, que en torno al 15 d.C. inician su producción en estas figlinae aretinas, como L.TI()A()CVArr 2122, L.TI()AG()CVArr 2123, L.TI()CE()CVArr 2124, L.TI()CRES()CVArr 2125, L.TI()FA()CVArr 2126, L. TI()FE(LIX)CVArr 2127, L.TI()GA()CVArr 2128, L.TI() HY()CVArr 2129, L.TI()MA()CVArr 2130, L.TI()OR() CVArr 2131, L.TI()PH()CVArr 2132, L.TI()CAS()CVArr 2133, L.TI()CLE() 2134, L.TI()CO()CVArr 2135, L.TI() CRE()CVArr 2136, L.TI()FL()CVArr 2138, L.TI()FO() CVArr 2139, L.TI()IA(NUARIVS) CVArr 2140, L.TI() N()CVArr 2141, L.TI()0()CVArr 2142, L.TI()SE()CVArr 2122, L.TI()SV()CVArr 2144, L.TI()VE()CVArr 2145.

Las piezas de L. Titivs han sido ampliamente documentadas en la península Ibérica en cartuchos in p.p., sin ser totalmente exhaustivos podemos citar los sigilla de este tipo hallados en el Tossal de Manises (Lucentum, Alicante) aunque sin cronología definida (Ribera 1988-1989: 178; Poveda 1996); Tarragona, Ampurias, Arragona (Sabadell), la Alcudia (Ilici, Elche) (Poveda 1996), Herrera de Pisuerga, Itálica, Castillones (Málaga), Celsa, Represas (Beja), Huesca (Beltrán 1990: 70); Segóbriga, Lugo o Bracara Augusta (Caamaño y López 2006: 84).

La utilización de piezas aretinas en ámbitos funerarios oretanos se ha documentado con anterioridad, en concreto en Laminium (Alhambra, Ciudad Real). En su necrópolis denominada Camino del Matadero fue hallado un plato en la misma posición funcional que el caso ahora presentado (Madrigal y Fernández Rodríguez 2001: 239). Junto a este plato, también aparecieron otros ejemplares que cubrían urnas funerarias de incineración en las tumbas 6,7 y 8 de esta misma necrópolis (ibidem). La existencia en esta necrópolis de elementos como los ungüentarios cerámicos y lacrimatorios puede ser reflejo de un ritual más romanizado que en el caso del Cerro de las Cabezas. Los excavadores de la necrópolis laminitana citan otros ejemplos de sigillatas aretinas sirviendo como tapaderas sobre urnas pintadas indígenas en necrópolis como las albacetenses de Mahora y Hoya de Santa Ana (ibidem: 241). También se conocen otras cerámicas romanas de paredes finas utilizadas como tapaderas de urnas pintadas de tradición indígena, como ilustra bien el de las que se hallaron en la necrópolis de los Ojos del Guadiana (Urbina y Urquijo, 2000).

En cuanto a la datación es posible señalar que, atendiendo a la tipología del plato, las producciones en esta forma se inician hacia el 15 a.C., prolongándose hasta el 40 d.C. Por otro lado, la cronología general de la Forma 20 se sitúa entre el 1-80 d.C. (Passelac 1993: 560). No obstante, las subformas 20.5.1 y 20.5.2 pueden datarse entre el 1-50 d.C., pues su producción pudo prolongarse en la etapa tardía de la TSI (50/60 d.C.) (Ettlinger et al. 1990: 86).

La presencia de la forma del cartucho in planta pedis y la disposición del mismo que presenta este plato, además de la aparición del uso del motivo decorativo aplicado en la superficie exterior, permiten fijar la producción en un momento posterior al 15 d.C. y hasta el cierre de la figlina de Lucius Titius, hacia el 40 d.C. (Poveda y Benítez de Lugo 2015, 8).

Es un momento en el cual el registro arqueológico es muy limitado en Oretania septentrional, desconociéndose en la mayoría de los casos los estratos testigos de la dialéctica inicial entre las comunidades prerromanas con los recién llegados. Son contadas las excepciones en las que se conoce que las antiguas ciudades ibéricas muestren alguna vitalidad en época altoimperial, como es el caso de Sisapo (La Bienvenida-Almodóvar del Campo, Ciudad Real), Laminium (Alhambra, Ciudad Real) o Libisosa (Lezuza, Albacete) (Zarzalejos et al. 2011: 34; Poveda 2002: 5-38; Uroz Sáez 2012: 116; Benítez de Lugo 2001; Benítez de Lugo et al. 2011). En el caso de la colonia foroaugustana no hay que olvidar que su territorium estaba política y económicamente vinculado con el de Carthago Nova, que por disponer de un importantísimo portus debió ser el probable punto de entrada del plato ahora encontrado. La llegada de este plato de sigillata itálica a Oretania pudo producirse en unos pocos poco tiempo después de su fecha de fabricación, a través de la Vía de los Vasos de Vicarello, tras cruzar Libisosa y Mentesa Oretana o el importante núcleo viario de Laminium (Benítez de Lugo et al. 2012a; Sánchez Sánchez et al. 2012).

Es preciso considerar que la triple moldura propia de la subformas 20.5 .1 y 20.5 2 comienza a introducirse hacia finales del reinado de Augusto, así como la decoración aplicada con motivos de dobles espirales, que son propias de finales de los tiempos de Augusto, encontrándose ya sólo muy generalizadas hacia el final del reinado de Tiberio. No obstante, en este momento se suele dotar 
a estas piezas evolucionadas de la Forma 18 -que copian a claros prototipos metálicos- de un mayor número de motivos aplicados que pueden oscilar entre 6 y 8 , además de aumentar el repertorio estilístico con series de delfines, máscaras, cuadrúpedos, que aparecen incluso intercalados en el propio vaso.

La pertenencia de esta pieza a una producción de L.Titivs la inscribe, por tanto, en ese momento tardío para el grupo de figuli que sellan mayoritariamente in planta pedis sus productos en Arretivm a partir del 15. d.C.

Por lo que respecta a la difusión de la Forma 20.5, ésta es documentada en múltiples enclaves del Mediterráneo y del interior de Europa. Sin ser exhaustivos podemos citar como ejemplos el caso de su hallazgo en Albintimilium, Annaba, Augsburg, Berenize, Corinto, Emona, Ephesos, Gubio, Knosos, Ordona, Ostia, Sirmium, Smyrna o Tipasa, o en la península Ibérica, en Béja (Ettlinger 2002: 86), Antic portal de Magdalena (Pérez 1990: 177. 197), Plaza Sant Joan (Lleida) (Junyent y Pérez 1994), Paeria (Lleida) (Pérez 1999: 222).

Dada la ausencia de alteración del enterramiento, el hecho de que no aparezca completo el plato de sigillata parece indicar que fue amortizado roto. Ello puede deberse a que ciertamente entero no guardaría la proporción armónica habitual que suelen tener estos conjuntos cerámicos funerarios ibéricos, en los que la pieza de cubierta se adapta casi a la perfección -o excede con ala muy limitada-al diámetro de la boca de la urna. Por otra parte, el hecho de que apareciera incompleta y rota en un contexto arqueológico cerrado podría implicar quizá la escasez de recursos económicos del finado.

La tumba descrita pertenece a la clase de enterramientos ibéricos adscritos la fase denominada de 'Romanización material', que se da en todo el evante peninsular durante la etapa republicana y, de forma algo más tardía, en la Meseta. Se trata de la primera fase de la romanización de los ritos funerarios iberos, caracterizada por la introducción en los ajuares de materiales romanos -por lo tanto de importación- dentro de contexto rituales que son básicamente indígenas. Es un momento de escasas modificaciones en la comprensión de lo funerario, lo cual indica que la sociedad indígena en estas fechas apenas ha reaccionado aún en lo que se refiere a las conductas e ideología -que tienen su reflejo social en las necrópolis- del ser humano ante la muerte. Para algunos autores, la verdadera normalización funeraria derivada de la romanización no llegará hasta el s. II d.C., con la generalización de las inhumaciones (Fuentes 1991: 589-590).
Sin embargo, es posible que una cosa sea la romanización cultual y otra la institucional. Es así porque Libisosa, uno de los oppida bien estudiados en Oretania septentrional, experimentó un cambio importante con su conversión en la colonia foroaugustana a la que Roma otorgó, tal y como se desprende de la lectura de Plinio el Viejo (HN III, 25), el ius italicum, la más alta consideración jurídica, quizás como premio para fijar la población en esta zona tan estratégica. Seguramente dicha promoción responde al programa político diseñado por Augusto, si bien lo habría aplicado poco después su sucesor, pues existen huellas materiales en los sondeos efectuados bajo los pavimentos de la curia y del edificio situado al $\mathrm{N}$ de la plaza forense que ofrecen una cronología del principado de Tiberio, además de que todos los hallazgos escultóricos son de fase tiberiana y posterior (Poveda et al. 2008, 481-497), igualmente se debe tener en cuenta que toda la epigrafía conocida hasta la fecha, tanto la inédita como la publicada (Abascal 1990: 43-49; Abascal y Sanz 1993: 23-26; Uroz Sáez 2012: 117-120; Poveda 2015) es de comienzos de Tiberio y posterior, quizá menos en un caso que no cambiaría el panorama epigráfico. La cuestión sobre la datación de la fundación de la colonia de Libisosa ha sido abordada recientemente teniendo en cuenta su contexto histórico (Uroz Rodríguez 2012: 22; Uroz Sáez 2012: 105 y ss.), que contrasta abiertamente con los datos arqueológicos citados que no pueden obviarse y por ello el mejor candidato para la fundación colonial sigue siendo Tiberio.

Por otra parte, en lo que a la romanización del Cerro de las Cabezas respecta, la existencia de este enterramiento y su necrópolis asociada implican, de forma evidente, la pervivencia de asentamiento en este lugar en fechas más recientes que el s. III a.C. El dato supone, por tanto, una contradicción con la propuesta de los excavadores del poblado, quienes relacionan un supuesto abandono del lugar con las incursiones cartaginesas en el interior de la Meseta (Vélez y Pérez 1987: 183). No es probable la postulada idea del arrasamiento, incendio y abandono repentino del Cerro de las Cabezas como explicación del final de sus habitantes, pues no existen evidencias de esas destrucciones en los perfiles estratigráficos ni tampoco en los contextos arqueológicos. Y sí, por el contrario, una contracción del asentamiento a partir del periodo Ibérico Reciente. Cerámicas campanienses y otras evidencias de poblamiento iberorromano, romano y medieval se encuentran al pie del Cerro de las Cabezas, de forma similar a lo sucedido en Cerro Domínguez, Giribaile y tantas otras ciudades prerromanas oretanas (Benítez de Lugo y Moraleda 2013: 251). 
En esta vieja ciudad oretana del Cerro de las Cabezas también se produjo un fenómeno nada extraño durante la romanización en Hispania: el desplazamiento de la población del oppidum al llano. Los oretanos del interior de la Meseta apenas aparecen citados en los textos de conquista romanos, lo que debe interpretarse como que estaban pacificados. No se conoce que el ambicioso programa romano de reordenación del territorio hispano, que incluyó la fundación de numerosas ciudades o la incorporación de otras antiguas como centros de comunicaciones o administrativos -véanse los casos de las vecinas Sisapo (La Bienvenida-Almodóvar del Campo, Ciudad Real), Laminium (Alhambra, Ciudad Real), Mentesa Oretana (Villanueva de la Fuente, Ciudad Real) o Libisosa (Lezuza, Albacete)-, alcanzara al antaño relevante oppidum del Cerro de las Cabezas. El cambio de era pudo ser para este lugar, con las evidencias disponibles, un momento de pérdida de pulso económico y político, si bien no de abandono y despoblamiento total. Desde esa ocupación romana y medieval en llano, los descendientes de los pobladores del Cerro de las Cabezas, habitantes de la Encomienda de Corral Rubio -así es como se llamo en época medieval el asentamiento situado a los pies del oppidum-, se enterraron en la misma vega en donde lo habían hecho sus antepasados, despoblando progresivamente a lo largo de la Edad Media el lugar para agruparse en otro más ventajoso, que sería denominado Valdepeñas (Benítez de Lugo et al. 2012b).

\section{CONCLUSIONES}

El mundo funerario del notable oppidum Cerro de las Cabezas (Valdepeñas, Ciudad Real) se conocía poco hasta ahora. Por el momento tan sólo se habían detectado algunos enterramientos puntuales dentro del poblado, pero no un área funeraria asociada al mismo.

El hallazgo de parte de una de sus necrópolis ha permitido, además de un avance en este campo, conocer más sobre la evolución del proceso de romanización de esta ciudad.

La Tumba 1 de la necrópolis del Cerro de las Cabezas es, de las catorce documentadas, la mejor conservada. Fue encontrada en un contexto arqueológico cerrado. Contiene los restos de una cremación enterrados en una urna -ollita pintada de tradición indígena- tapada mediante un plato de terra sigillata aretina, cuya forma
Consp. 20.5.2 es inédita en los repertorios arqueológicos de la provincia de Ciudad Real. No se puede descartar que otras de las tumbas identificadas hayan perdido algunas posibles tapaderas también de cerámica sigillata, pero su estado de mayor destrucción ha impedido certificarlo.

La incorporación funcional al rito funerario indígena de otros platos de sigillata aretina había sido documentada antes en otros contextos arqueológicos oretanos, como es la necrópolis de Alhambra. Sin embargo, el caso ahora presentado resulta novedoso, además de por la forma de la que se trata, por el hecho de encontrarse sellado. La pieza cuenta con una marca de alfarero in planta pedis con leyenda L.TITI, también inédito en la provincia de Ciudad Real. Es la primera ocasión en que en Oretania se documenta una cartela asociada a este alfar que produce sellando con el nomen de L. Titivs.

Nuestro plato aretino se puede datar, como se ha dicho, en un arco cronológico comprendido entre el 15/37 y el 40 d.C.

Este enterramiento con ritual ibérico que incorpora materiales de importación supone la pervivencia del poblamiento en el entorno del Cerro de las Cabezas a comienzos de nuestra era. El patrón de asentamiento no permaneció inmutable, naturalmente, desde la Edad del Bronce en que comienza la ocupación de este enclave. En efecto, en la actualidad no se conocen evidencias de que la poderosa ciudad ibérica consiguiera durante la romanización mantener su pujanza y dinamismo. La continuidad en el asentamiento que se conoce para los casos de las vecinas Laminium, Mentesa Oretana, Libisosa o Sisapo, parece haber funcionado de forma diferente en el caso del Cerro de las Cabezas. La limitada superficie excavada del oppidum no ha revelado por el momento indicios fehacientes de romanización, que sí aparecen en su necrópolis y a los pies de la ciudad. En el futuro será preciso investigar otras zonas del yacimiento, así como sus alrededores, para conocer los procesos de cambio y transformación vividos aquí al final de la Protohistoria.

En definitiva, la Tumba 1 de la necrópolis del Cerro de las Cabezas viene a significar y llamar la atención sobre la continuidad del poblamiento en el lugar, la incorporación de platos aretinos a los ritos funerarios ibéricos en sustitución de otros de barniz rojo, de barniz negro áticos y campanienses, y la perpetuación del ancestral rito funerario ibérico de incineración durante los primeros años de nuestra era en el $\mathrm{S}$ de la Meseta. 


\section{AGRADECIMIENTOS}

Enrique Mata Trujillo, arqueólogo, Diego Torres Marín e Ignacio Torres García participaron en la excavación de esta necrópolis Rocío Noval Clemente fue codirectora de estas excavaciones arqueológicas. Félix González González y Álvaro Rodríguez González autorizaron los trabajos arqueológicos en esta parcela y dieron todas las facilidades posibles para el desarrollo de los mismos.

\section{BIBLIOGRAFÍA}

ABASCAL, J. M. (1986): La cerámica pintada de tradición indígena en la Península Ibérica. Centros de producción comercio y tipología, Madrid.

ABASCAL, J. M. (1990): Inscripciones romanas de la provincia de Albacete, Albacete.

ABASCAL, J. M.; SANZ, R. (1993): Novedades de epigrafía romana en la provincia de Albacete, Al-Basit, 33, 13-36.

BELTRÁN, M. (1990): Guía de la cerámica romana, Zaragoza.

BENÍTEZ DE LUGO, L. (2001): El registro arqueológico en Lamimium-Alhambra (Ciudad Real), Cuadernos del Instituto de Estudios Manchegos 23-24, 9-25.

BENÍTEZ DE LUGO, L.; ÁLVAREZ GARCÍA, H. J.; FERNÁNDEZ MONTORO, J. L.; MATA, E.; MORALEDA, J.; SÁNCHEZ SÁNCHEZ, J.; RODRÍGUEZ MORALES, J. (2012a): Estudio arqueológico en la Vía de los Vasos de Vicarello A Gades Romam, entre las estaciones de Mariana y Mentesa (Puebla del Príncipe, Villanueva de la Fuente, Ciudad Real, AEspA 85, 101-108.

DOI: https://doi.org/10.3989/aespa.085.012.006

BENÍTEZ DE LUGO, L.; ANGULO, M. I.; DÍAZ BRAVO, J.; MATA, E.; MORALEDA, J.; PALOMARES, N.; SÁNCHEZ GARCÍA, J.; SÁNCHEZ SÁNCHEZ, J.; TORRES, M. (2012b): Los orígenes de Valdepeñas (Ciudad Real): el vicus romano y despoblado medieval de Aberturas. Investigación histórica y arqueológica, Munibe $63,255-291$

BENÍTEZ DE LUGO, L.; CABRERA, I.; RUIZ GÓMEZ, P.; MATA, E. (2011): Arqueología Urbana en Alhambra (Ciudad Real). Investigaciones sobre Laminium, Puertollano.

BENÍTEZ DE LUGO, L.; ESTEBAN, G.; HEVIA, P. (2004): Protohistoria y Antigüedad en la provincia de Ciudad Real (800 a.C.-500 d.C.), Ciudad Real.

BENÍTEZ DE LUGO, L.; MORALEDA, J. (2013): Símbolos, espacios y elementos ibéricos para el culto en Oretania septentrional. Estado de la cuestión arqueológica, revisión crítica y nuevas aportaciones, Congreso Internacional Santuarios iberos: territorio, ritualidad y memoria. El Santuario de la Cueva de La Lobera de Castellar (Jaén) 1912-2012 (C. Rísquez, C. Rueda, eds.), 213-270.
ETTLINGER, E.; HEDINGER, B.; HOFFMAN, B.; ROTH-RUBI, K.; KENRICK, P. M.; PUCCI, G.; SCHNEIDER, G.; SCHNURBEIN, S. VON; WELLS, C.M.; ZABEHLICKY SCHFFENEGGER, S. (1990): Conspectus formarum terrae sigillatae Italico modo confectae, Materialien zur römisch-germanischen Keramik 10, Bonn.

FERNÁNDEZ OCHOA, C.; ZARZALEJOS, M. M. (1991): Las producciones de terra sigillata Alto imperial de Sisapo (La Bienvenida, Ciudad Real). Terra sigillata Itálica y Gálica, Espacio Tiempo y Forma 6, Serie II, 255-280.

FERNÁNDEZ RODRÍGUEZ, M.; SERRANO, A. (1993): Una necrópolis iberorromana en Laminium (Alhambra, Ciudad Real), Revista de Arqueología 112, 46-53.

FUENTES, A. (1991): La fase final de las necrópolis ibéricas, Congreso de Arqueología Ibérica: Las Necrópolis. Serie Varia I, 587-606, Madrid.

HOFMANN, B. (1985): Catalogue des estampilles sur vaisielle sigilleé, Revue Archéologique Sites Hors-Série 27.

LÓPEZ PÉREZ, M. C.; CAAMAÑO, J. M. (2006): Adenda al corpus de marcas de alfarero en terra sigillata localizadas en Galicia, Gallaecia 25, 83-129.

JUNYENT, E.; PÉREZ FERNÁNDEZ, A. (1994): Los restos arqueológicos de la plaza de Sant Joan de Lleida I, RAP 4 173-203.

MADRIGAL, A.; FERNÁNDEZ RODRÍGUEZ, M. (2001): La necrópolis ibérica del Camino del Matadero (Alhambra, Ciudad Real), Arqueología funeraria: las necrópolis de incineración (F. J. Morales Hervás, R. García Huerta, coords.), 225-258, Universidad de Castilla-La Mancha.

MUNSELL, C. (2004): Munsell soil color Charts, Munssell Color, GretagMacbeth, New Windsor, NY.

OLCESE, G. (2011-2012): Atlante dei siti di produzione ceramica (Toscana, Lazio, Campania e Sicilia), Roma.

OXE, A.; CONFORT, H.; KENRICK, PH. (2000): Corpus vasorum arretinorum. A catalogue of the Signatures, Sharpes and Cronology of Italian Sigillata, Bonn.

PASSELAC, M. (1993): Céramique sigillée italique, DICOCER1, Lattara 6, 555-568.

PÉREZ FERNÁNDEZ, A. (1990): La terra sigillata de l'Antic Portal de Magdalena (Lleida), Monografies d'Arqueología Urbana 1, Lleida.

PÉREZ FERNÁNDEZ, A. (1999): Las excavaciones de la Paeria, Lleida. Los niveles augusteos y bajo-imperiales: La terra sigillata, RAP 9, 215-252.

POVEDA, A. M. (1996): La Terra Sigillata y el comercio romano en Contestania, Tesis Doctoral, Universidad de Alicante.

POVEDA, A. M. (2002): Fora Hispana. La evidencia de Libisosa Forum Augustum (Lezuza, Albacete), Conimbriga 41, 5-38. DOI: https://doi.org/10.14195/1647-8657_41_1

POVEDA, A. M.; UROZ, J.; MUÑOZ, J. (2008): Hallazgos escultóricos en la colonia romana de Libisosa (Lezuza, Albacete), Escultura romana en Hispania V (J.M. Noguera, E. Conde, eds.), 481-497. 
POVEDA, A. M. (e.p.): De epigrafía libisosana. Identificación de inscripción con nuevos datos sobre la presencia de la gens Maxvma, Actas de I Reunión Científica de Arqueología de Albacete (Albacete 22-23 de enero de 2015).

POVEDA, A. M.; BENÍTEZ DE LUGO, L. (2015): Nuevo ejemplo del uso de Sigillata Itálica en rituales funerarios oretanos. La Tumba 1 del oppidum Cerro de las Cabezas (Valdepeñas Ciudad Real), Boletín Ex Officina Hispana 6, 6-9.

PUCCI, G. (1985): Atlante delle forme ceramiche, II, Roma.

RIBERA, A. (1988-1989): Marcas de TS del Tossal de Manises, Lucentum VII-VIII, 171-204

SÁNCHEZ SÁNCHEZ, J.; BENÍTEZ DE LUGO, L.; RODRÍGUEZ MORALES, J.; FERNÁNDEZ MONTORO, J. L. (2012): Nomenclatura viaria Antigua. La Vía de los Vasos de Vicarello: una Vía Augusta en Hispania, El Nuevo Miliario $15,3-21$.

URBINA, D.; URQUIJO, C. (2000): La necrópolis íbero-romana de Los Toriles-Casas Altas (Villarrubia de los Ojos), El Patrimonio Arqueológico en Ciudad Real. Métodos de trabajo y actuaciones recientes (L. Benítez de Lugo, coord.), Valdepeñas, 153-166.
URBINA, D.; URQUIJO, C.; MORÍN, J.; TAPIAS, F. (2013): Madrigueras II: Un vicus en el territorio segobricense, Madrid.

URBINA, D.; URQUIJO, C.; BENÍTEZ DE LUGO, L. (2015): La romanización a través de las necrópolis de incineración en el entorno de Daimiel. Contextos Arqueológicos e inferencias culturales dentro el Área Manchega a partir de Los Toriles-Casas Altas (Villarrubia de los Ojos), Laminium (Alhambra) y Cerro de las Cabezas (Valdepeñas), III Jornadas de Historia de Daimiel, Daimiel, 45-59.

UROZ RODRÍGUEZ, H. (2012): Prácticas rituales, iconografía vascular y cultura material en Libisosa (Lezuza, Albacete). Nuevas aportaciones al Ibérico Final del Sudeste, Alicante.

UROZ SÁEZ, J. (2012): La colonia romana de Libisosa y sus precedentes, La ciudad romana en Castilla-La Mancha (G. Carrasco, coord.), 87-130. Universidad de Castilla-La Mancha.

VÉLEZ, J.; PÉREZ AVILÉS, J. (1987): El yacimiento protohistórico del Cerro de las Cabezas (Valdepenas), Oretum III, 167-196.

ZARZALEJOS, M.; FERNÁNDEZ OCHOA, C.; HEVIA, P. (2011): Investigaciones arqueológicas en Sisapo, capital del cinabrio hispano (I). La decoración musivaria de la domus de las Columnas Rojas (La Bienvenida-Almodóvar del Campo, Ciudad Real), Madrid. 